Nenko Radeff. L'élevagə du buffle en Bulgarie. Revue d'Histoire Natur. appl., p. 257, 1928.

Georg Tartler. Di甲 Büffelmilch. Zeitschr. für Fleisch und Milch-Hygiene, 1918 , S. 327.

\title{
LE DOSAGE DES CHLORURES DANS LES LAITS (1)
}

\author{
par
}

\section{A. MASSOT et H. LESTRA}

Dans un article de MM. Raquet et KeRLEveo (2) l'attention est attirée sur le fait qu'il est difficile, pour des raisons diverses, d'obtenir des chiffres exacts dans le dosage des chlorures du lait.

L'utilisation des cendres aboutit à des pertes dues à la volatilité des chlorures.

Le procédé de Denigès au métaphosphate, rigoureusement suivi entraîne des erreurs dues au fait que le métaphosphate permet bien un virage au jaune de l'alun de fer en présence de sulfocyanate, mais empêche, ou du moins retarde considérablement l'apparition de la teinte caractéristique rouge, persistante, marquant la fin de la réaction. Les chiffres de chlorures obtenus par ce procédé sont toujours trop faibles.

Notre article a pour but de proposer : $1^{\circ}$ des modifications rendant utilisables deux procédés classiques ; $2^{\circ}$ une technique originale de dosage des chlorures du lait.

\section{Méthode de Denigès modifiée}

Le liquide clair provenant de la défécation au métaphosphate est soumis à une ébullition de cinq minutes après addition d'azotate d'argent et d'acide nitrique. Dans ces conditions le métaphosphate est transformé en phosphate qui ne gêne pas le virage de l'alun de fer par le sulfocyanate. D'autre part, le chlorure d'argent est coagulé par l'ébullition et n'a pas tendance, comme le précipité plus ou moins colloïdal obtenu à froid, à réagir sur le sulfocyanate ferrique en le décolorant (3).

En résumé la méthode à utiliser est la suivante :

Dans un matras jaugé de. $100 \mathrm{~cm}^{3}$, introduire $10 \mathrm{~cm}^{3}$ de lait, $3 \mathrm{~cm}^{3}$ de solution de métaphosphate à $5 \%, 60$ à $70 \mathrm{~cm}^{3}$ d'eau, agiter, puis ajouter $10 \mathrm{~cm}^{3}$ d'acide sulfurique $\mathrm{N} / 10$, compléter à $100 \mathrm{~cm}^{3}$

(1) Extrait du Bull. Scien. Pharmacolojiques, 37e année, 1935, no 10. - Annales des Falsifications et des Fraudes, no 324 , déce mbre 1935 , p. 608.

(2) D. Raquet et A. Kerleveo. Dosage des chlorures dans le lait, Annales des Falsifications, $23^{\ominus}$ année, 1927. p. 580.

(3) Treadwell. Manuel de chimie analytique. I. Analyses quantitatives, 1925, p. 659. - Falsifications, $\mathrm{n}^{\circ} 324$. 
avec de l'eau distillée, agiter, filtrer, recueillir $58 \mathrm{~cm}^{3} 5$ de liquide clair, ajouter 5 à $6 \mathrm{~cm}^{3}$ d'acide nitrique, $5 \mathrm{~cm}^{3} \mathrm{NO}^{3} \mathrm{AgN} / 10$. Porter à l'ébullition 5 minutes. Laisser refroidir. Ajouter de l'alun de fer puis du sulfocyanate de potasse N/10 jusqu'à virage au rose persistant.

\section{Destruction permanganique}

La méthode de LAUDAT appliquée dans les conditions opératoires précisées ci-dessous, donne un liquide clair, où seule la matière grasse n'est pas minéralisée et où le virage de l'alun de fer se produit nettement.

A $10 \mathrm{~cm}^{3}$ de lait ajouter dans l'ordre $5 \mathrm{~cm}^{3}$ de solution $\mathrm{N} / 10 \mathrm{NO}^{3}$ $\mathrm{Ag}, 20 \mathrm{~cm}^{3}$ de solution saturée de permanganate de potasse. Porter à l'ébullition en agitant.

Au liquide chaud ajouter $40 \mathrm{~cm}^{3}$ d'acide nitrique pur.

Porter de nouveau à l'ébullition et maintenir celle-ci jusqu'à obtention d'un liquide clair.

Dans ces conditions la destruction est rapide et, dans le deuxième temps, il n'y a pas à craindre de mousse abondante entraînant des pertes.

Comme on le voit, la réussite de l'opération (et nous aurons l'occasion d'y revenir au sujet du plasma et des globules sanguins) tient aux proportions relatives de matière organique à détruire, de permanganate et d'acide nitrique.

Il est important d'introduire la liqueur argentique avant addition du permanganate, l'oxydation pouvant entraîner des pertes de chlore gazeux s'il n'y a pas d'argent pour fixer cet halogène.

De plus, l'attaque des matières albuminoïdes est plus rapide si on laisse agir, d'abord le permanganate, sans acide nitrique, ce dernier coagulant les albuminoïdes et rendant de ce fait leur attaque par les oxydants beaucoup plus difficile.

Enfin le-virage sera très net si on opére l'addition de sulfoeyanate sur les liqueurs refroidies - le chlorure d'argent étant, d'autre part, coagulé par l'ébullition.

\section{Technique originale}

\section{(Défécation à l'alcool acétone)}

Préparer le liquide précipitant suivant :

Alcool dénaturé (exempt de chlore) . . . . . . . . . . . . 3 parties Acétone ............................. 1 partie

Dans une fiole jaugée de $100 \mathrm{~cm}^{3}$ introduire environ $60 \mathrm{~cm}^{3}$ d'alcool-acétone et ajouter goutte à goutte $10 \mathrm{~cm}^{3}$ de lait ; compléter à $100 \mathrm{~cm}^{3}$ avec l'alcool-acétone. 
Filtrer sur un filtre plissé, en repassant les premières liqueurs ; le liquide s'écoule clair.

Recueillir $75 \mathrm{~cm}^{3}$ de liquide filtré, auquel on ajoute de l'acide nitrique $5 \mathrm{~cm}^{3} ; 5 \mathrm{~cm}^{3}$ d'azotate d'argent $\mathrm{N} / 10$ et de l'alun de fer.

Enfin, faire des affusions de liqueur décinormale de sulfocyanate jusqu'à virage au rouge - soit $n \mathrm{~cm}^{3}$ de sulfocyanate $\mathrm{N} / 10$ utilisés : $\frac{5-n \times 0,00585 \times 100 \times 4}{3}=$ chlorures exprimés en $\mathrm{NaCl}$ par litre de lair.

Nous nous réservons de faire connaître l'application de cette méthode au dosage du chlore sanguin.

Par cette méthode, sans intervention de la chaleur, on obtient un virage très net de l'alun de fer en présence de sulfocyanate et la précision est égale à celle de n'importe quel autre procédé ainsi qu'on peut s'en rendre compte par le tableau ci-dessous.

\begin{tabular}{c|c|c|c}
\hline $\begin{array}{c}\text { Méthode de Denigès } \\
\text { au } \\
\text { métaphosphate }\end{array}$ & $\begin{array}{c}\text { Méthode de Denigès } \\
\text { au métaphosphate } \\
\text { modifiée }\end{array}$ & $\begin{array}{c}\text { Destruction } \\
\text { permanganique }\end{array}$ & $\begin{array}{c}\text { Défécation } \\
\text { à } \\
\text { l'alcool acétone }\end{array}$ \\
\cline { 2 - 3 } 0,76 & 1,52 & 1,52 & 1,58 \\
1,10 & 2,17 & 2,20 & 2,14 \\
1,05 & 1,52 & 1,46 & 1,53 \\
0,98 & 1,80 & 1,74 & 1,75 \\
0,87 & 1,78 & 1,75 & 1,71 \\
0,90 & 1,43 & 1,44 & 1,46 \\
0,98 & 1,57 & 1,54 & 1,58 \\
\hline \hline
\end{tabular}

La méthode la plus rapide, donnant le virage le plus net, est celle qui utilise l'alcool-acétone comme déféquant.

Les deux autres méthodes, utilisant la chaleur, exigent un refroidissement qui est toujours assez long et qui est cependant indispensable à la netteté du virage.

\section{BIBLIOGRAPHIE ANALYTIQUE}

\section{LES LIVRES}

Besana (G.) et Del Guerra (M.). - Sottoprodotti del Latte e loro utilizzatione (Sous-produits du lait et leur utilisation). Vol. III : Enciclopedia Del Caseificio. Un vol. 432 p., 105 fig., relié, 18 lires. Editeur : Ulrico Hoepli, Milano, 1936.

Ce troisième volume de l' "Enciclopedia del Caseificio" a été composé avec la même allure que les volumes antérieurs : sur le "Lait ", sur " Le Beurre 